\title{
Early prediction of cause-specific acute respiratory distress syndrome via interpretable machine- learning.
}

\section{Junwei Wu}

General Hospital of People's Liberation Army: Chinese PLA General Hospital

Chao Liu ( $\square$ liuchao5186@gmail.com )

Ping An Healthcare Technology https://orcid.org/0000-0002-6708-4654

\section{Lixin Xie}

General Hospital of People's Liberation Army: Chinese PLA General Hospital

\section{Xiang Li}

Ping An Healthcare Technology

\section{Kun Xiao}

General Hospital of People's Liberation Army: Chinese PLA General Hospital

\section{Guotong Xie}

Ping An Technology

\section{Fei Xie}

General Hospital of People's Liberation Army: Chinese PLA General Hospital https://orcid.org/00000003-3674-8216

\section{Research Article}

Keywords: early prediction, cause-specific, intensive care units, ARDS (acute respiratory distress syndrome), interpretable machine learning, time-phased.

Posted Date: March 22nd, 2021

DOl: https://doi.org/10.21203/rs.3.rs-323879/v1

License: (c) (i) This work is licensed under a Creative Commons Attribution 4.0 International License. Read Full License 
1 Early prediction of cause-specific acute respiratory distress syndrome via interpretable machine learning

4 Authors' names and affiliations

5 Junwei $\mathrm{Wu}^{1, *}$, Chao $\mathrm{Liu}^{2, *}$, Lixin, $\mathrm{Xie}^{3,{ }^{3,}}$, Xiang $\mathrm{Li}^{2}$, Kun $\mathrm{Xiao}^{3}$, Guotong Xie ${ }^{2,4,5, \#}, \mathrm{Fei} \mathrm{Xie}^{3, \#}$

$6 *$ These authors contributed equally to this work.

$7 \quad{ }^{1}$ Library of Graduate School, Chinese People's Liberation Army General Hospital, 100853

8 Beijing, China

$9 \quad{ }^{2}$ Ping An Healthcare Technology

$10{ }^{3}$ Department of Pulmonary and Critical Care Medicine, Chinese People's Liberation Army

11 General Hospital, 100853 Beijing, China.

$12 \quad{ }^{4}$ Ping An Health Cloud Company Limited

13 5Ping An International Smart City Technology Co., Ltd.

14 \# Corresponding author

Corresponding authors

17 Guotong Xie Email: xieguotong@pingan.com.cn \& Fei Xie: xiefei0522@163.com 


\section{Abstract}

Background: Several studies have investigated the correlation between physiological parameters and the risk of acute respiratory distress syndrome (ARDS); however, causespecific ARDS and its early prediction have not been well-studied. We aimed to develop and validate a machine-learning model for the early prediction of inhalation-induced ARDS.

Methods: Clinical expertise was applied with data-driven analysis. Using data from electronic intensive care units (retrospective derivation cohort) and the three most accessible vital signs (i.e. heart rate, temperature, and respiratory rate) together with feature engineering, we applied a random forest approach during the time window of 90 hours that ended 6 hours prior to the onset of moderate-to-severe respiratory failure (the ratio of partial pressure of arterial oxygen to fraction of inspired oxygen $\leqslant 200 \mathrm{mmHg}$ ).

Results: The trained random forest classifier was validated using an independent validation cohort, with an area under the curve of 0.9026 (95\% confidence interval $0.8075-1)$ and optimal threshold probability, the accuracy, sensitivity, and specificity values were $0.8947,1$, and 0.875 , respectively. A Stable and Interpretable RUle Set (SIRUS) was used to extract rules from the predictive ability for inhalation-induced ARDS and may assist clinicians in decision-making, as well as facilitate the enrolment of patients in prevention programmes to improve their 
outcomes.

Key words: early prediction, cause-specific, intensive care units, ARDS (acute respiratory distress syndrome), interpretable machine learning, time-phased.

\section{Background}

Acute respiratory distress syndrome (ARDS) is life-threatening and the major cause of morbidity and mortality in intensive care units (ICUs), with a mortality rate exceeding $40 \%$ [ 1 , 2]. The high public health impact of ARDS is due to the paucity of effective therapies [3]. Therefore, early prediction of patients with a high risk for developing ARDS and the use of prevention strategies for such patients are of great value in critical care units [4]. However, healthcare providers experience challenges in recognising ARDS patients [5], which may be due to the diversity of causes (e.g. inhalation, trauma, coronavirus disease [COVID-19]) [4]. Additionally, ARDS is rarely present at the time of hospital admission $[5,6]$, increasing the risk of clinicians not readily recognising, interpreting, and acting upon relevant information [7].

Because of the high mortality and difficulty in disease recognition, understanding the relationship between risk factors and ARDS is of considerable value. For example, serum zinc levels have been recommended as an appropriate biomarker for evaluating the risk of developing inhalation-induced ARDS [8]. Additionally, XGBoost, an open-source software library, has been adopted to identify ARDS based on non-invasive physiological parameters, such as heart rate and respiratory rate [9]. Alternatively, machine-learning has recently been used for the early prediction of ARDS [5]. Although ARDS prediction has attracted the attention of many researchers, previous studies have been focused on all-cause ARDS, without considering the aetiology of the disease, and studies on cause-specific ARDS are lacking. In 
addition, it is challenging to translate machine-learning methods into clinical practice owing to the following reasons: 1) most models have not been validated using an independent test set and therefore have unknown generalisability [10]; 2) ensemble learning models (e.g. XGBoost), despite providing good prediction performance, lack transparency (interpretability); and 3) many models incorporate too many indices (e.g. laboratory blood tests), increasing their potential complexity and burden in clinical practice [5, 9-11].

To overcome the above obstacles, we focused on inhalation-induced ARDS. Owing to the nature of the injury (e.g. smoke from fires, smoke bombs), patients with inhalation-induced ARDS often present in groups, increasing pressure on clinical practitioners. Limited access to health care resources, such as low nurse-to-patient ratios, has made the situation worse; for example, it has caused delays in initiating and providing adequate treatment for deteriorating patients and higher ICU mortality [7]. Thus, this study aimed to develop a simple transparent (interpretable) model to predict early inhalation-ARDS with generalisability using data from eICUs [12], as a retrospective derivation cohort, and independently validate the model with freely available critical care data from the Medical Information Mart for Intensive Care III (MIMIC-III; www.mimic.physionet.org) [13]. Our model is able to reliably predict the onset of moderate-to-severe inhalation-induced ARDS 6 hours prior to onset.

\section{Methods}

This study used a random forest approach for the early prediction of inhalation-induced ARDS. To develop the model, we used a retrospective observational cohort, obtained from the eICU Collaborative Research Database that consists of inpatient ICU encounters at 32 de-identified medical centres between 2014 and 2015. Nine patient encounters from the MIMIC-III database were used as a validation cohort. Both databases are publicly accessible, and the MIMIC-III 
publication states that 'the project was approved by the Institutional Review Boards of Beth Israel Deaconess Medical Center (Boston, MA) and the Massachusetts Institute of Technology (Cambridge, MA)'. Requirement for individual patient consent was waived because the project did not impact clinical care and all protected health information was deidentified.

Trained investigators extracted data from electronic patient medical records from both cohorts. Selection of patients was based on ICD-9/10 codes and diagnostic key words. Patients with previous ICU admission were excluded. Each patient was cared for at a single medical centre. We applied additional inclusion criteria to focus the scope of our study. Patient stays that did not involve at least one recorded measurement of the variables were excluded $[5,11]$. In addition, we included only patient stays with a duration within a specified window (see "Predictor variables" for details). The final derivation cohort (i.e. eICU) consisted of five unique patients corresponding to 48 unique observations. The final validation cohort (i.e. MIMIC-III) comprised three unique patients corresponding to 19 observations. The characteristics of both cohorts are presented in Table 1. Supplemental Figure 1 outlines the patient selection process and details the number of patients after each selection procedure.

\section{Outcome variables}

The primary outcome of interest in this study was the ratio of partial pressure of arterial oxygen to fraction of inspired oxygen $\left(\mathrm{PaO}_{2} / \mathrm{FiO}_{2}\right.$ ratio $) 6$ hours since investigation. ARDS was diagnosed according to the Berlin definition established in 2012 [14]. Depending on the severity of lung failure according to the $\mathrm{PaO}_{2} / \mathrm{FiO}_{2}$ ratio, ARDS is currently classified as mild $\left(200<\mathrm{PaO}_{2} / \mathrm{FiO}_{2} \leqslant 300\right)$, moderate $\left(100<\mathrm{PaO}_{2} / \mathrm{FiO}_{2} \leqslant 200\right)$, or severe $\left(\mathrm{PaO}_{2} / \mathrm{FiO}_{2} \leqslant 100\right)$ $[14,15]$. In the model development, the numeric ratio was converted to a binary outcome: presence of moderate or severe ARDS $\left(\mathrm{PaO}_{2} / \mathrm{FiO}_{2} \leqslant 200\right)$ vs. mild or better ARDS 
128 Table 1 Characteristics of the final cohort

129

\begin{tabular}{|c|c|c|c|}
\hline & $\begin{array}{c}\text { Derivation cohort } \\
\text { eICU }\end{array}$ & $\begin{array}{l}\text { Validation cohort } \\
\text { MIMIC-III }\end{array}$ & $\mathrm{p}$-value \\
\hline $\begin{array}{l}\text { \# Data points } \\
\text { (Subjects N) }\end{array}$ & $48(5)$ & $19(3)$ & \\
\hline Age, years & $42.2(31.0-57.6)$ & $63.3(48.6-84.6)$ & 0.22 \\
\hline Females & $1(5)$ & $0(3)$ & 1 \\
\hline $\begin{array}{c}\text { Hospital discharge } \\
\text { status-death }\end{array}$ & $2(5)$ & $0(3)$ & 0.86 \\
\hline Ethnicity, N (\%) & & & \\
\hline White & $4(80 \%)$ & $2(66.67 \%)$ & \\
\hline Hispanic & $1(20 \%)$ & 0 & \\
\hline Others (unknown) & 0 & $1(33.33 \%)$ & \\
\hline Length-of-stay & 321.82 & 85.48 & \\
\hline (LOS) hours & 44.13 & 181.48 & \\
\hline (Difference & 91.95 & 91 & 1 \\
\hline between admission & 849.53 & & \\
\hline and discharge) & 4.17 & & \\
\hline
\end{tabular}

130 Data are presented as mean (95\% range) or N (proportion), unless otherwise stated. 
For model development, the predictive power of three primary vital signs (body temperature, pulse (heart rate $[\mathrm{HR}]$ ), and breathing rate (respiratory rate)), were evaluated. These were selected as predictor variables because they are the most accessible, non-invasive physiological parameters that are continually monitored. In addition, measurements for these vital signs generated in different platforms are easily compared as they are not affected by external factors or obtained by means of different medical devices. Thus, if a correlation between the vital signs and ARDS could be established, it would allow a prediction with simplicity and generalisability.

Feature engineering was performed before features were included in the random forest analysis.

We adopted a "time-phased" strategy that calculated the minimum, maximum, and mean values of the three vital signs collected during a $90 \mathrm{~h}$ time window beginning $96 \mathrm{~h}$ prior to the onset of ARDS and ending $6 \mathrm{~h}$ prior to the onset from every recorded blood gas test (by which the $\mathrm{PaO}_{2} / \mathrm{FiO}_{2}$ ratio could be calculated) [16]. The feature's spatiotemporal pattern representation is shown in Supplemental Figure 2 for two representative patients from each cohort.

\section{Model development}

We adopted a random forest classifier, an ensemble learning method for classification, to predict the binary diagnosis outcome of ARDS. In the random forest analysis, each tree was constructed using a different bootstrap sample from the original training data [17]. The parameter "Number of trees" was set to 5,000 to ensure that every input row would be predicted at least a few times; all other parameters were set to default. During the initial model training process, nine predictor variables during the 90-hour time window were included: minimum, maximum, and mean HR; minimum, maximum, and mean respiratory rate; and minimum, maximum, and mean temperature. The importance of each variable was ranked according to 157 the mean decrease in Gini values. The top four features (i.e. resp_96h_6h_mean, 
HR_96h_6h_mean, resp_96h_6h_min, and temp_96h_6h_max) were finally selected because their importance, measured according to the mean decrease in Gini value, was higher than the average mean decreases in Gini for all candidate features. In this way, we could halve the number of features in the model for a small cost in accuracy.

\section{Statistical analysis}

Cohort characteristics were expressed as mean (range) and proportions (n, \%), as dictated by the data type. We used the t-test and Wilcoxon test to compare mean values of continuous variables, and $\chi^{2}$ test to compare the frequencies of categorical variables between two groups. Gini-based feature selection is implemented in the randomForest $\mathrm{R}$ package [18].

Receiver operating characteristic (ROC) curves were generated to quantify the predictive accuracy of the models, and the area under the curve (AUC) was used to assess the discriminatory ability of the models. Cut-off threshold for optimal point was determined by performing ROC curve analysis in the retrospective cohort [6].

Data were analysed using R V.4.0.1. Two-sided p values $<0.05$ were considered statistically significant.

\section{Results}

\section{Performance on independent test set}

The binary ARDS diagnosis prediction performance is summarised using a ROC curve in Supplemental Figure 3. The classifier demonstrated an area under the ROC curve (AUROC) of 0.9026 (95\% confidence interval [CI] 0.8075-1) for early ARDS prediction with the independent test set (MIMIC-III) 6 hours prior to the onset of moderate or severe ARDS. The 
corresponding ROC curve is shown in Supplemental Figure 3.

We also measured the prediction performance of the model using other evaluation metrics (i.e. sensitivity, specificity, and accuracy), and using a decision curve analysis. To address the class imbalance in the critical care outcome (i.e. the low proportion of moderate or severe conditions), we chose the threshold of prediction results based on the ROC curve (i.e. the value with the shortest distance to the perfect model) $[19,20]$. With an optimal threshold probability of 0.65 , the accuracy, sensitivity, and specificity values were $0.8947,1$, and 0.875 , respectively. Thus, the decision curve analysis provided insight into avoiding unnecessary interventions for patients with a low likelihood of moderate or severe ARDS onset.

\section{Model interpretation}

The lack of interpretability of the ensemble learning methods is a strong limitation for applications involving clinical decision-making. To overcome this issue, we employed an interpretable random forest model, Stable and Interpretable RUle Set (SIRUS) [21], as implemented in the R package SIRUS [22], to extract rules from a tree ensemble based on their frequency of appearance. As stated in the original method article "the most frequent rules, which represent robust and strong patterns in the data, are ultimately linearly combined to form predictions" [23]. SIRUS outputs a simple set of 10 rules (with all parameters set to default). To generate the prediction for each specific instance, SIRUS considers whether the conditions for each rule are satisfied to assign one of the two possible likelihood output values. In the next step, the 10-rule outputs were averaged to provide the predicted probability of the onset of moderate or severe ARDS. The 10-rule set is presented in Table 2. Overall, the rules, generated by the interpretable model, provide transparent and concise information regarding how the prediction is made, thus overcoming the aforementioned challenges hindering translational 
research of ARDS.

\section{Discussion}

211 In this study, we developed a machine-learning model for the early prediction of inhalation212 induced ARDS (moderate-to-severe condition) at 6 hours prior to onset in critical care units.

213 The model demonstrated good prediction performance and clinical interpretability on eICU data. Specifically, the model achieved a high AUROC with an independent validation set (i.e. MIMIC-III). All features used in the model were derived from clinical variables that are routinely collected in ICUs, which can be simply and non-invasively obtained. This model can be easily applied to ICU patients to assist clinical decision-making and thus, holds great potential for improving patient outcomes.

Importantly, the model provided a convenient way of calculating the likelihood of the target event, i.e. the development of inhalation-induced ARDS. To the best of our knowledge, no previous study has used the SIRUS in a clinical setting. The model improved prediction performance based on the random forest approach while maintaining transparency. More precisely, the rule set and how each of the rules contributed to the prediction was clearly shown. Instead of a decision-making path (where rules work interactively), those rules work independently towards a probability, making mechanistic exploration and/or explanation more realistic. 
233 Table 2 Rules for calculating the likelihood of moderate to severe ARDS developing in six 234 hours

\begin{tabular}{|c|c|c|c|}
\hline No. & Conditions & Satisfied & $\begin{array}{c}\text { Not } \\
\text { satisfied }\end{array}$ \\
\hline 1 & resp_96h_6h_min $<9$ & 0.864 & 0.269 \\
\hline 2 & resp_96h_6h_mean $<16.1$ & 0 & 0.684 \\
\hline 3 & HR_96h_6h_mean $<102$ & 0.447 & 0.9 \\
\hline 4 & temp_96h_6h_max $<100$ & 0.292 & 0.792 \\
\hline 5 & temp_96h_6h_max $<100$ & 0.222 & 0.733 \\
\hline 6 & resp_96h_6h_mean $<19$ & 0.211 & 0.759 \\
\hline 7 & $\begin{array}{c}\text { resp_96h_6h_mean } \geq 16.1 \& \\
\text { resp_96h_6h_min } \geq 9\end{array}$ & 0.412 & 0.613 \\
\hline 8 & HR_96h_6h_mean $<84.3$ & 0.333 & 0.636 \\
\hline 9 & $\begin{array}{c}\text { resp_96h_6h_mean } \geq 16.9 \& \\
\text { resp_96h_6h_min }<9\end{array}$ & 0.905 & 0.259 \\
\hline 10 & HR_96h_6h_mean $<110$ & 0.488 & 1 \\
\hline
\end{tabular}

236 To generate the predicted likelihood of the presence of moderate to severe acute respiratory 237 distress syndrome (ARDS), the model considers whether the conditions for each rule are 238 satisfied to assign one of the two possible output values. For example: resp_96h_6h_min $=5: 0$ 239 (minimum value of respiratory rate of all available respiratory rate measured during 96-6 hours 240 prior to the target time point), then the first rule is satisfied, returning $p(1)=0.864$. Next, the 24110 rule outputs are averaged to provide the predicted probability of ARDS (moderate or severe) 242 onset. 
244 There are several vital signs typically examined by medical professionals, such as temperature, $\mathrm{HR}$, and respiratory rate. Additionally, although blood pressure is not considered a vital sign, it is often measured alongside the vital signs. However, the three vital signs are generally more accessible and accurate than blood pressure, which is more prone to influence from external factors [24]. Moreover, model simplicity can lead to higher feasibility of implementation, particularly in low-income countries that lack ICU capacity. Furthermore, because of infrequent vital sign monitoring and a lack of standardised management practices in resourcelimited settings, basic monitoring measures need to be better used [25]. Thus, we included only three simple, non-invasive, routinely monitored vital signs in our model.

The risk factors identified in our study are consistent with those from previous studies and were validated using an independent validation set. In 2020, Liu et al. compared the most commonly used early warning scoring systems for hospitalised patients with and without infection at risk for in-hospital mortality and transfer to the ICU and found that the National Early Warning Score (NEWS) exhibited the highest discrimination for mortality (followed by the Modified Early Warning Score-MEWS) [26]. The main findings of the present study agreed with the role of the NEWS [27]. For example, a respiratory rate $\leqslant 9$ breaths/min increases the risk of developing critical or moderate-to-severe ARDS according to both the NEWS and our model.

Rather than providing a binary outcome, our model is able to calculate the likelihood of developing moderate-to-severe ARDS. This was achieved by aggregating the vital sign measurements collected during hospital stay to yield the score used in our model, allowing the output to reflect risk for the target event. In addition, our prediction leaves a definite time window prior to the event, making potential intervention realistic. Such time-phase determination is suitable for clinical practice as clinical practitioners generally prefer having a 
suitable time window that is neither too far nor too near to onset, in order to leave sufficient time for intervention. The exact time window for feature engineering can be treated as a hyperparameter, which may vary slightly across different cohorts (e.g. patient characteristics) [16]. However, parameter tuning was outside the scope of this study.

In comparison with other similar studies using machine-learning models for the early prediction of ARDS, our model showed improved prediction performance. The prediction performance measured using the AUROC ranged from 0.75 to 0.87 in previous studies $[5,28$, 29], whereas our model achieved an AUROC of 0.9026 with an independent validation set. In addition, the predictors used in our model were further simplified [5]. A potential explanation may be that the retrospective cohort included in this study were cause-specific ARDS patients (ARDS patients with inhalation injury). To validate the model further, we applied the model to our own clinical data from one inhalational ARDS patient with 61 data points (number of moderate or severe ARDS instances: 46) and achieved an AUROC of 0.84 (95\% CI: $0.7465-$ 0.9347) (Ethics approval can be found in the supplementary materials).

Although the prediction ability of the model was validated using two completely unrelated data sets, our study had some limitations. Similar to the limitations in previous data challenges "the analysis was performed in a retrospective setting. The generalisability and stability of the proposed model need to be evaluated systematically in prospective settings" [16]. Moreover, we included first admissions only, which might be a potential source of selection bias. We also acknowledge the limitation that the sample size was small; however, we included 48 data points with each class label being balanced during model derivation. The model was further validated in an independent external data set (i.e. MIMIC-III). We believe that studies with a small number of subjects can quickly address research questions in a relatively short space of time; 
thus, a more efficient allocation of resources (e.g. subjects, time, financial costs) will be

295

296

297

298

299

300

301

302

303

304

305

306

307

308

309

310

311

312

313

314

315

316

317

318 achieved by first testing a new research hypothesis in a small number of subjects [30]. We also reviewed similar studies and found that for some diseases with a rare prevalence and incidence, such as spinal cord injury, small sample sizes were employed in 167/207 (81\%) studies and the median total sample size was 18 (range 4-582) in published research [31]. Furthermore, the random forest-based classifier fits studies with a small sample size well, for example, data sets characterised by an extremely large $\mathrm{p}$ (number of features)/n (number of instances) ratio in genomic data analysis [32], and a study on the desired sample size for a desired model accuracy was also presented [33].

\section{Conclusions}

In this study, an interpretable machine-learning model with key features (derived from three non-invasively measured vital signs) was successfully established for predicting inhalationinduced ARDS 6 hours prior to onset. The prediction model is intended to improve communication between nursing staff and junior doctors and "flag" patients who need to be given immediate priority [34]. Inhalation-induced ARDS typically affects individuals such as fire fighters and soldiers, who are often in otherwise good condition without chronic diseases, and providing critical care expertise at an early stage is extremely important for improving patient outcomes.

In future research, we hope to include more patients, while expanding to other cause-specific ARDS, such as COVID-19, as the current ARDS predictions for patients with COVID-19 are either risk factor analysis [35], real-time risk scoring systems [36], and/or utilising relatively complex predictors, such as the neutrophil-to-lymphocyte ratio [37]. We hope to accomplish this aim by integrating this early prediction model in our own ICU risk management system to 
evaluate its effectiveness.

320

321 In conclusion, as clinical instances accumulate and clinical records become more 322 comprehensive, this study forms a basis for evaluating the effectiveness of personalised 323 intervention (e.g. vital sign-directed therapy) [38]. Cause-specific medicine is a critical component of precision, personalised healthcare and will be the core of effective care in the anticipatable future.

\section{List of abbreviations}

328 ARDS: acute respiratory distress syndrome

329 AUC: area under the curve

330 CI: confidence interval

331 HR: heart rate

332 ICUs: intensive care units

333 MEWS: Modified Early Warning Score

334 MIMIC-III: Medical Information Mart for Intensive Care III

335 NEWS: National Early Warning Score

336 RF: random forest

337 ROC: Receiver operating characteristic

338 SIRUS: Stable and Interpretable Rule Set

Declarations

341 Ethics approval and consent to participate:

342 The Ethics Committee of Chinese PLA General Hospital approved the study and use of data 343 (Approval No. of Ethics Committee: S2020-532-02). 
345 The information of "consent for publication" can be found in the Approval Certificate of 346 Ethical Review form (Approval No. of Ethics Committee: S2020-532-02).

Availability of data and materials:

The data that support the findings of this study are available from Chinese PLA General Hospital, but restrictions apply to the availability of these data, which were used under license for the current study, and so are not publicly available. Data are however available from the authors upon reasonable request and with permission of Chinese PLA General Hospital.

\section{Competing interests:}

Chao Liu's, Drs. Li's, and G. Xie's institutions received funding from Big Data Research \&

Development Project of Chinese PLA General Hospital (Grant No. 2017MBD-011). The remaining authors have no conflicts of interest to disclose.

Funding:

360

This study was supported by the following funds and projects:

1.National Natural Science Foundation of China (61976223);

2.Medical Innovation Research Project of Chinese PLA General Hospital(2019YFC0121703);

3.Translational Medicine of Chinese PLA General Hospital (2018TM-03);

4.Clinical Support Project of Chinese PLA General Hospital (2018FC-WJFWZX-2-04); 011). 
369 FX and GX conceived the idea and supervised the study. JW contributed to the acquisition of 370 data; CL, XL analysed data and performed statistical data analysis; JW, CL, LX, XL, KX

371 contributed to the drafting of the manuscript; All authors reviewed and approved the final 372 version.

374 Acknowledgements:

375 We would like to thank Editage [http://www.editage.com] for editing and reviewing this 376 manuscript for English language. 


\section{References}

378

379

380

381

382

383

384

385

386

387

388

389

390

391

392

393

394

395

396

397

398

399

400

401

1. Villar J, Blanco J, Añón J, Santos-Bouza A, Blanch L, Ambrós A, et al. The ALIEN study: incidence and outcome of acute respiratory distress syndrome in the era of lung protective ventilation. Intensive Care Med. 2011.

2. Reyna MA, Josef CS, Jeter R, Shashikumar SP, Sharma A. Early Prediction of Sepsis From Clinical Data: The PhysioNet/Computing in Cardiology Challenge 2019. Critical Care Medicine. 2019;48(2):1.

3. Zhang R, Wang Z, Tejera P, Frank AJ, Wei Y, Su L, et al. Late-onset moderate to severe acute respiratory distress syndrome is associated with shorter survival and higher mortality: a two-stage association study. Intensive care medicine. 2017;43(3):399-407.

4. Luo J, Yu H, Hu Y-H, Liu D, Wang Y-W, Wang M-Y, et al. Early identification of patients at risk for acute respiratory distress syndrome among severe pneumonia: a retrospective cohort study. Journal of thoracic disease. 2017;9(10):3979.

5. Le S, Pellegrini E, Green-Saxena A, Summers C, Hoffman J, Calvert J, et al. Supervised machine learning for the early prediction of acute respiratory distress syndrome (ARDS). Journal of Critical Care. 2020;60:96-102.

6. Trillo-Alvarez C, Cartin-Ceba R, Kor DJ, Kojicic M, Kashyap R, Thakur S, et al. Acute lung injury prediction score: derivation and validation in a population-based sample. European Respiratory Journal. 2011;37(3):604-9.

7. Hyland SL, Faltys M, Hüser M, Lyu X, Merz TM. Early prediction of circulatory failure in the intensive care unit using machine learning. Nature Medicine. 2020;26(3):364-73.

8. Xie F, Zhang X, Xie L. Prognostic value of serum zinc levels in patients with acute HC/zinc chloride smoke inhalation. Medicine. 2017;96(39).

9. Yang $\mathrm{P}, \mathrm{Wu} \mathrm{T}$, Yu M, Chen F, Wang C, Yuan J, et al. A new method for identifying the acute respiratory distress syndrome disease based on noninvasive physiological parameters. 
PloS one. 2020;15(2):e0226962.

10. Ding X-F, Li J-B, Liang H-Y, Wang Z-Y, Jiao T-T, Liu Z, et al. Predictive model for acute respiratory distress syndrome events in ICU patients in China using machine learning algorithms: a secondary analysis of a cohort study. Journal of translational medicine. 2019;17(1):1-10.

11. Le S, Pellegrini E, Green-Saxena A, Summers C, Hoffman J, Calvert J, et al. Supervised Machine Learning for the Early Prediction of Acute Respiratory Distress Syndrome (ARDS). medRxiv. 2020.

12. Pollard TJ, Johnson AE, Raffa JD, Celi LA, Mark RG, Badawi O. The eICU Collaborative Research Database, a freely available multi-center database for critical care research. Scientific data. $2018 ; 5(1): 1-13$

13. Johnson AE, Pollard TJ, Shen L, Li-Wei HL, Feng M, Ghassemi M, et al. MIMIC-III, a freely accessible critical care database. Scientific data. 2016;3(1):1-9.

14. Force ADT, Ranieri V, Rubenfeld G, Thompson B, Ferguson N, Caldwell E. Acute respiratory distress syndrome. Jama. 2012;307(23):2526-33.

15. Hernández-Beeftink T, Guillen-Guio B, Villar J, Flores C. Genomics and the Acute Respiratory Distress Syndrome: Current and Future Directions. International Journal of Molecular Sciences. 2019;20(16).

16. Li X, Xu X, Xie F, Xu X, Sun Y, Liu X, et al. A Time-Phased Machine Learning Model for Real-Time Prediction of Sepsis in Critical Care. Critical Care Medicine. 2020;48(10):e884-e8. 17. Yang F, Lu W-h, Luo L-k, Li T. Margin optimization based pruning for random forest. Neurocomputing. 2012;94:54-63.

18. RColorBrewer S, Liaw MA. Package 'randomForest'. University of California, Berkeley: Berkeley, CA, USA. 2018.

19. Kuhn M, Johnson K. Applied predictive modeling: Springer; 2013. 

Network Open. 2019;2(1).

21. Bénard C, Biau G, Veiga SD, Scornet E. SIRUS: Stable and Interpretable RUle Set for classification. Electronic Journal of Statistics. 2021;15(1):427-505.

22. Bénard C, Biau G, Da Veiga S, Scornet E. SIRUS: Stable and Interpretable RUle Set. 2020.

23. Bénard C, Biau G, Da Veiga S, Scornet E. Interpretable random forests via rule extraction. arXiv preprint arXiv:200414841. 2020.

24. Sapra A, Malik A, Bhandari P. Vital Sign Assessment- PMID: 319859942019.

25. Cummings MJ, Wamala JF, Bakamutumaho B, Davis JL. Vital signs: the first step in prevention and management of critical illness in resource-limited settings. Intensive Care Medicine. 2016;42(9):1-2.

26. Liu VX, Lu Y, Carey KA, Gilbert ER, Afshar M, Akel M, et al. Comparison of early warning scoring systems for hospitalized patients with and without infection at risk for inhospital mortality and transfer to the intensive care unit. JAMA network open. 2020;3(5):e205191-e.

27. Physicians RCo. National Early Warning Score (NEWS) 2: Standardising the assessment of acute-illness severity in the NHS. Updated report of a working party. London: RCP2017. 28. Taoum A, Mourad-Chehade F, Amoud H. Early-warning of ARDS using novelty detection and data fusion. Computers in biology and medicine. 2018;102:191-9.

29. Zhou M, Sharma R, Zhu H, Li Z, Fan X. Rapid breath analysis for acute respiratory distress syndrome diagnostics using a portable two-dimensional gas chromatography device. Analytical and Bioanalytical Chemistry. 2019;411(24):6435-47. 
31. Zimmermann G, Bolter LM, Sluka R, Hller Y, Bathke AC, Thomschewski A, et al. Sample sizes and statistical methods in interventional studies on individuals with spinal cord injury: A systematic review. Wiley-Blackwell Online Open. 2019;12(3).

32. Chen X, Ishwaran H. Random forests for genomic data analysis. Genomics. 2012;99(6):323-9.

33. Floares A, Ferisgan M, Onita D, Ciuparu A, Calin G, Manolache F. The smallest sample size for the desired diagnosis accuracy. Int J Oncol Cancer Ther. 2017;2:13-9.

34. Gardner-Thorpe J, Love N, Wrightson J, Walsh S, Keeling N. The value of Modified Early Warning Score (MEWS) in surgical in-patients: a prospective observational study. The Annals of The Royal College of Surgeons of England. 2006;88(6):571-5.

35. Xu W, Sun N-N, Gao H-N, Chen Z-Y, Yang Y, Ju B, et al. Risk factors analysis of COVID19 patients with ARDS and prediction based on machine learning. Scientific reports. 2021;11(1):1-12.

36. Liang M, He M, Tang J, He X, Liu Z, Feng S, et al. Novel risk scoring system for predicting acute respiratory distress syndrome among hospitalized patients with coronavirus disease 2019 in Wuhan, China. BMC infectious diseases. 2020;20(1):1-10.

37. Ma A, Cheng J, Yang J, Dong M, Liao X, Kang Y. Neutrophil-to-lymphocyte ratio as a predictive biomarker for moderate-severe ARDS in severe COVID-19 patients. Critical Care. 2020;24(1):1-4.

38. Hvarfner A, Blixt J, Schell CO, Castegren M, Baker T. Vital Signs Directed Therapy for the Critically Ill: Improved Adherence to the Treatment Protocol Two Years after Implementation in an Intensive Care Unit in Tanzania. 2020. 


\section{Supplementary Files}

This is a list of supplementary files associated with this preprint. Click to download.

- SupplementalFigure1.ThePatientSelectionProcess.pdf

- SupplementalFigure2.SpatiotemporalPatternRep..pdf

- SupplementalFigure3.ROCMIMICIII.pdf

- STROBEchecklistcohortfilled.docx 